\title{
Alcune idee per una riforma del SSN
}

\author{
Elio Borgonovi
}

Negli ultimi cinque-sei anni oltre 100 Paesi di tutto il mondo hanno messo mano in misura più o meno profonda ai propri sistemi di tutela della salute. Tali interventi, tuttavia, sono stati prevalentemente motivati da ragioni di non sostenibilità economica già attuale ma soprattutto proiettata nel medio-lungo periodo. Le proiezioni sono del tutto affidabili in quanto si basano sui trend demografici che consentono di prevedere la popolazione al 2030-2050, cui sono applicati indicatori di carattere epidemiologico, che rispondono al principio della evidence-based. Un altro elemento, meno prevedibile, che sta alla base di queste proiezioni/previsioni è quello del progresso scientifico nel campo della genomica, delle tecnologie mediche, delle nanotecnologie, della robotica applicata all'assistenza da cui comunque deriveranno enormi avanzamenti nel campo della qualità dell'assistenza, ma altrettanto sicure pressioni sul piano della spesa.

Si tratta quindi di una situazione generalizzata che presenta due principali tratti comuni: da un lato si focalizza l'attenzione sulla sostenibilità economico-finanziaria senza considerare la sostenibilità sociale, politica, istituzionale, dall'altro si affrontano separatamente (in genere due a due) le tendenze della domanda, dell'offerta, del finanziamento. Si tratta di due limiti derivanti dalla logica "riduttivistica" che ha caratterizzato il XX secolo, ma che è diventata dominante e sempre più forte negli ultimi 20-30 anni. Una logica basata sul piano economico dall'idea/illusione di una crescita lineare e illimitata (messa in discussione dalla crisi scoppiata nel 2007/2008 e dalla drammatica situazione dell'Europa a seguito del referendum greco del 5 luglio) e da quella di poter affrontare problemi complessi tramite la segmentazione e la separazione delle varie parti. Anche le riflessioni degli ultimi anni relative al SSN italiano sono state dominate da questa logica riduttivistica che, di volta in volta, ha portato al centro del dibattito i temi delle inaccettabili differenze tra Regioni (sono note le considerazioni sui 21 sistemi sanitari regionali), degli interventi per evitare eccessi di domanda (considerazioni sul ruolo della prevenzione e dell'appropriatezza), della lotta agli sprechi e del recupero di efficienza (considerazioni sulla medicina difensiva, sulle differenze nei costi di acquisto di beni simili, sui costi unitari dell'assistenza, sulla lotta alla corruzione), dei nuovi modelli assistenziali (considerazioni sulla de-ospedalizzazione, sui modelli per intensità delle cure, sull'assistenza territoriale, sulla continuità delle cure e l'organizzazione a rete, sulla presa in carico dei pazienti), delle fonti integrative di finanziamento (addizionali regionali, ticket $e$ altre forme di co-payment, fondi integrativi, casse mutue, in generale finanziamento garantito per cosiddetto secondo pilastro del welfare).

Questa logica riduttivistica ha portato a interventi caratterizzati da vincoli sui livelli di spesa (come esempio per tutti valgano gli interventi per le Regioni sotto piano di rientro e le norme sul blocco del turnover per le ultime leggi di stabilità) e da azioni frammentarie che, nella maggior parte dei casi, hanno aumentato la rigidità della gestione. Negli ultimi dieci anni si contano ormai a decine questi interventi disorganici e in molti casi contraddittori, il cui effetto è stato quello del contenimento del tasso di aumento della spesa sanitaria a fronte, però, di una crescente divaricazione tra i livelli assistenziali previsti formalmente da leggi nazionali e regionali e quelli effettivamente garantiti, tra livelli assistenziali di diverse Regioni o di diversi territori all'interno della stessa Regione, e della crescente insoddisfazione 
da parte di gruppi sempre più vasti di popolazione. Altrettanto frammentari sono stati gli interventi riconducibili al cosiddetto secondo pilastro del welfare e quelli dei fondi integrativi. Interventi che hanno sofferto della mancanza di organicità sia sul piano normativo, sia su quello del trattamento fiscale. Di fronte a questa crescente frammentarietà, soprattutto negli ultimi cinque/sei anni, è diventata sempre più forte l'esigenza di un ripensamento strutturale del servizio sanitario nazionale che, per essere utile, deve abbandonare "ideologie" di carattere politico-sociale (la difesa di principi astratti di "universalità, equità, non discriminazione, accessibilità" interpretati in astratto), economico ( fiducia nei sistemi di competizione tra strutture di offerta, di separazione tra finanziatori e fornitori di servizi mediati dal mercato o da quasi-mercati), istituzionale (fiducia nel valore cogente delle norme e della riallocazione dei poteri tra Stato e Regioni). A quasi quarant'anni dall'introduzione del SSN, poco oltre i vent'anni dalla riforma definita come aziendalizzazione (D.Lgs. 502/92 e 517/93) e a quindici da quella definita come "terza riforma rimasta in buona parte in mezzo al guado" (D.Lgs. 229/99) si impone uno sforzo di innovazione di carattere sociale e istituzionale che possa dare basi solide a una azione politica e a soluzioni economiche e organizzative realmente sostenibili per il medio-lungo periodo.

Un ripensamento che deve partire dalla riflessione sui principi fondamentali del SSN. Innanzitutto quello di universalità, che dà concretezza alla tutela della salute come diritto della persona sancito da dichiarazioni internazionali e dalla carta costituzionale. È ovvio che essa non può essere e non è mai stata intesa come "dare tutto a tutti" ma non può essere nemmeno intesa come diritto subordinato a vincoli di carattere economico. Per dare una soluzione concreta a quanto affermato dalla Corte Costituzionale, secondo cui diritto alla salute e i vincoli della finanza pubblica sono principi di pari dignità costituzionale, occorre scindere due aspetti di solidarietà che sottendono il principio di universalità. Il primo può essere definito come solidarietà obbligatoria di tipo fiscale, in base al quale $i$ soggetti a più alto reddito contribuiscono a sostenere le spese per la salute anche dei soggetti a più basso reddito. Il secondo consiste nella solidarietà "volontaria", che può essere realizzata tramite casse e fondi integrativi, società di mutuo soccorso liberamente scelte nell'ambito dei contratti collettivi (fondi chiusi) o lanciati sul mercato (fondi aperti). La distinzione che occorre mantenere è tra casse e fondi che si ispirano a principi solidaristici, ossia che non escludono persone sulla base del rischio individuale, che non differenziano i contributi secondo rischi di salute e che hanno finalità non profit, dalle assicurazioni che definiscono polizze di copertura riferite a specifici rischi individuali trattati collettivamente, ossia tenendo conto della numerosità dei sottoscrittori.

Questa distinzione consente di superare lo schema dei cosiddetti "fondi DOC" ai sensi del D.Lgs. 229/99, in applicazione di quanto già previsto dal D.Lgs. 502/92, quelli che godono di trattamento fiscale agevolato perché garantiscono solo prestazioni aggiuntive e integrative a quelle dei LEA, e altri fondi che non godono di questo trattamento, poiché garantiscono prestazioni che "teoricamente" e "astrattamente" sono già garantite dal SSN. Sono stati usati non casualmente i termini "teoricamente" e "astrattamente", poiché è stato ampiamente dimostrato da numerose indagini e ricerche che i livelli assistenziali effettivi sono molto differenziati tra Regioni, l'ottenimento delle prestazioni formalmente garantite è condizionato dalle lunghe liste di attesa e, in molti casi, dal fatto che gli ultimi mesi dell'anno le Aziende Sanitarie e le Aziende Ospedaliere hanno utilizzato il budget assegnato e quindi rinviano le prestazioni. Inoltre si ricorda che l'aggiornamento dei LEA è continuamente rinviato, soprattutto a causa di vincoli di compatibilità finanziaria, in quanto l'inclusione di nuovi trattamenti e di nuove tecnologie comporterebbe una pressione sui livelli di spesa. In un certo senso, negli ultimi 10-15 anni, è stata preferita quella che può essere definita una specie di "ipocrisia istituzionale e politica" che determina un razionamento di fatto dei livelli assistenziali e che aumenta le disuguaglianze di tipo territoriale e di tipo socio-economico, poiché non si è voluto introdurre un sistema di definizione esplicita di priorità nella allocazione delle risorse. Il superamento della distinzione tra fondi DOC e fondi non DOC è diventato sempre più ideologico, poiché non rispecchia la realtà attuale fortemente condizionata dai vincoli di finanza pubblica. Sarebbe quindi meglio un ripensamento del principio di universalità che preveda anche la libertà e possibilità per i privati di decidere riguardo all'allocazione del proprio reddito disponibile dopo la tassazione. Concedere un trattamento fiscale agevolato a soggetti (individui, famiglie, imprese) che ritengono prioritario allocare parte della propria ricchezza alla tutela della salute invece che ad altri consumi (auto, abbigliamento, sport, tempo libero ecc.) 
consentirebbe diversi vantaggi: un afflusso di risorse aggiuntive all'intero sistema della salute non derivante prevalentemente dalla spesa out-of-pocket (anomalia italiana rispetto ai Paesi avanzati), ma intermediata da soggetti che perseguono finalità di interesse per la comunità; spinte alla razionalizzazione del sistema di offerta che possono essere date da fondi, casse e mutue, oltre che dalle imprese di assicurazioni for profit, nei confronti di strutture private accreditate o anche di strutture pubbliche (es. accordi per l'uso dell'attività professionale intra moenia); riduzione della pressione sul sistema pubblico, in quanto parte della domanda troverebbe altri canali di offerta.

Il superamento della distinzione tra fondi DOC e fondi non DOC significa lasciare a soggetti privati la scelta sul tipo di bisogni cui essi intendono dare risposte. In particolare, essi potranno scegliere tra la garanzia di bisogni a elevata frequenza e a basso costo unitario, bisogni a frequenza relativamente bassa e con costi unitari medi o medio-alti e bisogni definibili di carattere catastrofico, il cui manifestarsi è difficilmente sopportabile dalla stragrande maggioranza della popolazione e potrebbe far cadere in povertà individui e intere famiglie. I bisogni del primo tipo possono essere soddisfatti completamente applicando il principio di solidarietà di fondi e casse integrative. I bisogni del secondo tipo possono essere coperti tramite un mix di intervento di fondi e casse di tipo solidaristico e di imprese professionalmente orientate a trattare questi tipi di rischi. I bisogni del terzo tipo possono essere soddisfatti prevalentemente da imprese di assicurazione o da fondi e casse che riescono a trovare soluzioni tecniche che integrano la copertura del rischio con l'elemento di solidarietà. La crisi del welfare state di tipo europeo ha messo in discussione il modello di universalismo monolitico calato/governato dall'alto. L'opzione da alcuni suggerita di opting out, ossia uscita dal SSN dei "ricchi che possono permettersi una assicurazione privata", è poco coerente con la cultura e la storia italiana perché porterebbe a un sistema "a doppia velocità", la sanità di qualità per pochi e quella di bassa qualità per molti, sistemi peraltro ben noti nell'esperienza degli USA, della Cina e di altri Paesi. L'evoluzione auspicabile è quella di un welfare "composito" $e$ "articolato", basato sulla solidarietà fiscale, solidarietà volontaria e l'assicurazione di rischi sanitari.

Una seconda linea di ripensamento riguarda l'assetto istituzionale, finalizzato a porre rimedio alla strutturale debolezza dell'attuale sistema, rappresentata dalla divaricazione tra i livelli assistenziali effettivamente garantiti dalle diverse Regioni. Moltissimi analisti ed esperti parlano esplicitamente di 21 sistemi sanitari regionali e non di un unico SSN. Occorre intervenire su questo punto tramite un'azione che può sembrare contraddittoria, poiché è necessario rafforzare il livello centrale senza indebolire il livello regionale. La contraddizione è solo apparente, poiché il mantenimento di un'autonomia delle Regioni sul piano della programmazione e dell'organizzazione dei servizi è finalizzato a dare risposte che meglio si adattano alle differenze storiche, culturali, geografiche economiche, sociali che sono presenti nel nostro Paese. Il rafforzamento del livello centrale è finalizzato a eliminare le differenze e le variabilità "negative" o non giustificabili, ossia derivanti da sprechi, inefficienze, corruzione e condizionamenti localistici. Per evitare approcci di tipo "modellistico", che significa proporre soluzioni costruite su una semplificazione del mondo reale, o di "ideologia di tipo istituzionale, economica e manageriale", che significa proporre soluzioni ottimali solo in astratto, è necessario chiarire bene quali sono le funzioni che si intende centralizzare e creare le condizioni affinché il livello centrale sia in grado di esercitare al meglio queste funzioni. Pertanto una riforma organica dovrebbe prevedere il rafforzamento delle seguenti funzioni a livello statale. Innanzitutto la definizione di un quadro legislativo che dia nuovi contenuti al diritto alla tutela della salute. Infatti, se con la 833/78 si è passati dal concetto di lotta alla malattia a quello di promozione della salute, oggi occorre passare al concetto di presa in carico della persona. Ciò significa enunciare principi che inducano le Regioni a introdurre modelli gestionali e organizzativi basati sulla maggiore integrazione tra ospedale e territorio e sulla reale continuità delle cure. In secondo luogo, la normativa dovrebbe fare qualche passo in avanti con riguardo all'interpretazione da dare alla sentenza della Corte Costituzionale, che ha dichiarato la parità del valore costituzionale da attribuire al principio di tutela della salute e a quello della salvaguardia dell'equilibrio della finanza pubblica.

In terzo luogo, la normativa dovrebbe definire in modo più chiaro ed esplicito l'interdipendenza tra politiche di tutela della salute, politiche nel campo socio-assistenziale e politiche di altri settori. Esistono molte evidenze secondo cui la prevenzione primaria si attua agendo su fattori quali gli stili di vita e l'ambiente, per cui l'efficacia dipende da politiche della famiglia, dello sport, del lavoro, dei trasporti, della tutela ambientale, della lotta al fumo e a tutte le dipen- 
denze. A sua volta la presa in carico delle persone che si trovano in situazioni di cronicità, che oggi sono la maggioranza e assorbono la maggior parte della spesa (alcuni studi stimano in circa $70 \%$ la spesa destinata a pazienti cronici), richiede interventi qualificabili come sanitari, socio-sanitari, socio-assistenziali e l'uso integrato di flussi finanziari che passano attraverso il canale del SSN, degli enti locali, degli enti previdenziali (INPS, INAIL ecc.). In quarto luogo, va superata anche una visione che tiene nettamente distinte le problematiche del SSN rispetto alle problematiche di politica industriale del nostro Paese. In passato spesso ha prevalso la logica secondo cui le politiche del SSN devono avere l'obiettivo di massimizzare il livello di salute e minimizzare la spesa (o i costi) per il SSN, senza preoccuparsi dell'impatto sui settori delle imprese collegate. Questa visione ha causato l'indebolimento di molte imprese italiane, per esempio la quasi scomparsa delle attività di ricerca svolte nel nostro Paese. Oggi appare sempre più evidente che occorre sostituire l'obiettivo di perseguire "soluzioni ottimali per un settore" (per esempio del SSN), per cercare invece di perseguire obiettivi di "ottimizzazione complessiva" della filiera collegata ai bisogni di salute, sia con riguardo alla domanda interna, sia con riferimento alle potenzialità di esportazione di farmaci, tecnologie e dispositivi medici, soluzioni di e-health ecc.

In quinto luogo, la normativa deve includere principi e criteri per assicurare la sostenibilità del SSN nel lungo periodo. Si tratta di esplicitare principi e regole decisionali finalizzati a raggiungere la sostenibilità economico-finanziaria (collegata al finanziamento pubblico e privato nelle diverse forme), ma anche quella sociale (livelli di assistenza effettivamente garantiti anche in rapporto alle attese suscitate dal progresso scientifico e dalla circolazione delle informazioni) e politica (intesa non tanto come consenso politico ma come conservazione di un elevato livello di legittimazione delle istituzioni). Ciò può essere ottenuto con logiche innovative di programmazione che abbia le seguenti caratteristiche:

- coerenza tra obiettivi, risorse finanziarie, strutturali e tecnologiche, dotazione di personale con le professionalità che si adeguano al mutamento delle conoscenze e delle tecnologie adottate;

- selettività, nel senso che occorre definire chiare priorità tra gli obiettivi;

- focalizzazione, che significa avere piani di poche decine di pagine evitando documenti corposi che pochi leggono e nei quali si disperdono le scelte rilevanti;

- individuazione di standard (appropriatezza, efficacia, qualità dei servizi, dotazione di strutture, efficienza, costo) intesi non come vincoli rigidi, ma come strumenti che impongono alle Regioni e alle strutture che erogano servizi di verificare il proprio posizionamento (positivo o negativo) e che consentono l' inversione della prova", ossia la possibilità di discostarsi dagli standard se si è in grado di fare meglio con soluzioni organizzative e gestionali diverse;

- flessibilità di tipo spaziale, ossia possibilità di valorizzare le "differenze fisiologiche" dei diversi territori e delle diverse comunità, e di tipo temporale, nel senso di scorrimento e possibilità di adattare criteri e standard al mutamento delle condizioni epidemiologiche, economiche, organizzative, tecnologiche ecc.

Peraltro, il rafforzamento del livello centrale con la finalità di armonizzare i livelli di risposta nei diversi ambiti territoriali non può limitarsi alla ricostruzione di un nuovo quadro giuridico. Esso deve riguardare anche il rafforzamento delle capacità di governo, indirizzo, monitoraggio della transizione. Deve essere superata la logica, che sempre diventa illusione, secondo cui una volta approvate nuove regole per il sistema esso possa conformarsi a queste regole. Occorre una logica proattiva, ossia di gestione del processo di cambiamento, che si proponga di esaltare le novita positive e ridurre al minimo le possibili distorsioni. A livello centrale dovranno essere garantite le seguenti condizioni:

- formazione di un gruppo significativo di esperti (almeno 200/300) dei cambiamenti istituzionali, ossia persone che abbiamo un mix di competenze tecniche (epidemiologiche, cliniche ecc.), organizzative, manageriali e di valutazione delle politiche pubbliche, che possano essere utilizzati come advisor delle Regioni (se richiesti, oppure imposti per le Regioni che dimostrano di non essere in grado di implementare il cambiamento); 
- ridefinizione della mission degli organi di supporto tecnico (ISS, Agenas e, parzialmente, AIFA), tramite la valorizzazione di competenze oggi esistenti e utilizzate in modo inadeguato, o la creazione di nuove competenze, come per esempio quelle specifiche di HTA o di modelli per la valutazione di politiche integrate di tutela della salute intesa ad ampio spettro;

- messa a punto di un sistema di indicatori in grado di cogliere con immediatezza i segnali positivi e quelli negativi relativi alla riforma del SSN;

- realizzazione di un database delle "buone pratiche", cosi numerose sul territorio italiano ma che oggi non vengono adeguatamente diffuse;

- attivazione o rafforzamento dei momenti di coordinamento delle diverse politiche nazionali che possono avere impatto sulla salute dei cittadini;

- attivazione di un flusso di investimenti pubblici e privati verso quella parte di economia reale rappresentata dalla filiera della salute, tramite soluzioni controllate di PPP.

Infine una provocazione sulla quale si invitano i lettori di questo editoriale a proporre reazioni e commenti da sottoporre alla Direzione di Mecosan. Molte leggi regionali già approvate o in corso di approvazioni hanno introdotto o prevedono di introdurre un livello di "programmazione, acquisto di servizi e controllo" distinto da quello di erogazione dei servizi. Ciò vuol dire avere quattro livelli di governo: quello centrale, quello regionale, quello sub-regionale di programmazione, acquisto e controllo, quello di erogazione dei servizi. Una catena decisionale indubbiamente pesante perfino per le imprese multinazionali o globali, figuriamoci per un sistema pubblico di tutela della salute. Se a ciò si aggiunge che gli istituti di ricovero e cura a carattere scientifico fanno già riferimento al livello centrale e che gli ospedali universitari hanno una loro autonomia, seppur coordinata con le politiche delle Regioni, si impone un ripensamento dell'assetto di governance complessivo per il quale si propongono alcuni principi. Innanzitutto, si potrebbe suggerire che il governo centrale definisca regole più chiare e precise di rapporti con le Regioni (nell'ambito della revisione del Titolo $V$ della Costituzione) e abbia maggiori poteri nel governo della rete di IRCCS e di grandi ospedali, che abbiano una rilevanza non solo territoriale e regionale, ma che si qualifichino come eccellenze a livello nazionale (e in alcuni casi internazionale), su cui concentrare investimenti strategici per l'intero SSN. Inoltre, per evitare che la definizione di LEA a livello nazionale si traduca poi in differenze sostanziali, dovrebbe essere rafforzata a livello centrale la funzione di definizione di standard strutturali, funzionali e professionali per le strutture che erogano servizi da applicare a quelle pubbliche, private o miste. Lo Stato dovrebbe porsi anche l'obiettivo di definire, oltre ai LEA, anche i livelli essenziali di organizzazione e dotazione di strutture in modo da ridurre la mobilità "inappropriata" che ha caratterizzato gli ultimi anni, in quanto, condizionato dalle differenti capacità delle Regioni, il SSN ha prodotto flussi di risorse dalle Regioni già deboli, in termini di qualità dell'offerta, alle Regioni già forti. Infine, un adeguato sistema di governance dovrebbe evitare che vi sia una logica a cascata, ma dovrebbe essere in grado di qualificare in modo preciso e puntuale le relazioni a due a due tra i quattro livelli. Dovrebbe quindi definire un quadro di riferimento per le relazioni tra Stato e Regione, Regioni e agenzie/aziende/ambiti di programmazione, acquisto e controllo, relazioni tra queste ultime e aziende pubbliche/private/miste che erogano i servizi. Nella definizione del sistema di governance dovrebbe poi essere affrontato in modo innovativo anche il tema dei medici dipendenti del Servizio Sanitario Nazionale, MMG e PLS, che oggi non sono dipendenti ma sono regolati da convenzioni nazionali. La continuità delle cure el'integrazione tra diverse fasi dell'assistenza (prevenzione, diagnosi e cura per acuti, assistenza post-acuzie e per pazienti cronici) impone di ricercare nuovi strumenti che favoriscano la collaborazione e l'integrazione tra medici, infermieri e altri professionisti oggi dipendenti del SSN e professionisti regolati da convenzioni. 